\title{
Network models of driver behavior
}

\author{
Markus T Mattsson Corresp. 1,2 \\ 1 Department of Psychology and Logopedics, University of Helsinki, Helsinki, Finland \\ 2 Traffic Research Unit, University of Helsinki, Helsinki, Finland \\ Corresponding Author: Markus T Mattsson \\ Email address: markus.mattsson@helsinki.fi
}

The way people behave in traffic is not always optimal from the road safety perspective: drivers exceed speed limits, misjudge speeds or distances, tailgate other road users or fail to perceive them. Such behaviors are commonly investigated using self-report-based latent variable models, and conceptualized as reflections of violation- and error-proneness. However, attributing dangerous behavior to stable properties of individuals may not be the optimal way of improving traffic safety, whereas investigating direct relationships between traffic behaviors offers a fruitful way forward. Network models of driver behavior and background factors influencing behavior were constructed using a large UK sample of novice drivers. The models show how individual violations, such as speeding, are related to and may contribute to individual errors such as tailgating and braking to avoid an accident. In addition, a network model of the background factors and driver behaviors was constructed. Finally, a model predicting crashes based on prior behavior was built and tested in separate datasets. This contribution helps to bridge a gap between experimental I theoretical studies and self-report-based studies in traffic research: the former have recognized the importance of focusing on relationships between individual driver behaviors, while network analysis offers a way to do so for self-report studies. 


\section{Network models of driver behavior}

2 Markus Tapani Mattsson ${ }^{1,2}$

$3{ }^{1}$ Department of Psychology and Logopedics, University of Helsinki, Helsinki, Finland

42 Traffic Research Unit, University of Helsinki, Helsinki, Finland

5

6 Corresponding Author:

7 Markus Mattsson ${ }^{1,2}$

8 Email address: markus.mattsson@helsinki.fi

9

10

11

12 


\section{Abstract}

The way people behave in traffic is not always optimal from the road safety perspective: drivers exceed speed limits, misjudge speeds or distances, tailgate other road users or fail to perceive them. Such behaviors are commonly investigated using self-report-based latent variable models, and conceptualized as reflections of violation- and error-proneness. However, attributing dangerous behavior to stable properties of individuals may not be the optimal way of improving traffic safety, whereas investigating direct relationships between traffic behaviors offers a fruitful way forward. Network models of driver behavior and background factors influencing behavior were constructed using a large UK sample of novice drivers. The models show how individual violations, such as speeding, are related to and may contribute to individual errors such as tailgating and braking to avoid an accident. In addition, a network model of the background factors and driver behaviors was constructed. Finally, a model predicting crashes based on prior behavior was built and tested in separate datasets. This contribution helps to bridge a gap between experimental / theoretical studies and self-report-based studies in traffic research: the former have recognized the importance of focusing on relationships between individual driver behaviors, while network analysis offers a way to do so for self-report studies.

\section{Introduction}

It has become something of a truism in human factors research that people contribute to road crashes by either deliberately violating rules or by making unintended errors. In traffic psychology, perceiving the importance of this distinction coincides with the development of a much-used questionnaire instrument, the Driver Behaviour Questionnaire ${ }^{1}$ (DBQ). A seminal study ${ }^{1}$ hypothesized that the distinction might be due to violations and errors being "mediated by different psychological mechanisms", and interpreted the results of a principal components "aberrant behavior" that need to be targeted by different types of intervention has since appeared in numerous research articles, some of which have been based on the errors / violations dichotomy, some of which have made finer distinctions between different types of errors and violations. Subsequent studies have commonly referred to Reason et al. ${ }^{1}$ as having proved the existence of two qualitatively different psychological mechanisms even though the authors of the original study were careful to note that the distinction was hypothetical in nature. The idea is echoed in statements such as: "Errors and violations result from different psychological processes (Reason et al., 1990) and therefore should be treated differently"2; "Since errors and violations result from different psychological processes, they should be treated differently (Reason et al., 1990)"3, and "As each type of behavior has a distinct psychological underpinning (Reason et al., 1990), different interventions are required to reduce their frequency and also associated crash risk"4 . This idea is herein referred to as the latent variable view of violations and errors. 
52 The received wisdom of the latent variable view has gone unchallenged for two main reasons.

53 First, a sufficiently detailed theory on the relationships between psychological mechanisms,

54 traffic behaviors and latent variables is still lacking. Second, individual violations and errors may

55 well be causally related: for example, exceeding the speed limit (a violation) may cause one to

56 miss observing something (an error). The present paper focuses on the latter issue, and

57 conceptualizes driver behavior in a novel manner as an interacting network of behaviors,

58 emotional reactions and perceptions. Similar network models have been recently employed in

59 research on psychopathology ${ }^{7,8}$, personality ${ }^{9}$, attitudes ${ }^{10}$ and intelligence ${ }^{11}$ as descriptions of how

60 the phenomena under investigation may arise from the interactions of their component parts.

61 Psychological mechanisms and latent variables in traffic psychology

62 Consider the above-cited assertion that dimensionality reduction produces insights about

63 psychological mechanisms. This is unlikely, since a mechanistic explanation of a cognitive

64 process involves accounting for the component operations of the process and their interaction ${ }^{12}$,

$65{ }^{13}$, while PCA and factor analysis (FA) are - stated simply - procedures for grouping correlated

66 variables. In fact, offering violation- and error-proneness as explanations for why individuals

67 break rules and commit errors amounts to reified circular reasoning ${ }^{14}$ insofar as the

68 "pronenesses" refer to nothing but being likely to behave in said manner. Furthermore, it has

69 been argued that a detailed analysis of psychological mechanisms should precede the generation

70 of psychometric instruments instead of being used as a post-hoc explanation for the correlational structure of the instrument in the data analysis stage ${ }^{68}$. When latent variable models have been constructed in this manner, based on theories of cognitive psychology, it has been observed that several psychological mechanisms may contribute to a given latent variable score ${ }^{15,68}$.

Further, the individual DBQ errors and violations are of such a wide variety that it is unclear how they could all be implemented by a single cognitive or psychological mechanism. For instance, the errors include missing an observation, forgetting the road one was travelling, ending up on an often-used route when intending to travel elsewhere etc. Accordingly, it has been argued that these errors are differentially related to top-down and bottom-up attention, updating of a mental model of the driving situation and resolving conflicts between schemas in memory 5 . Similar considerations apply to individual violations. Network models, with their focus on the interactions between traffic behaviors, may prove useful in practice in encouraging the researcher to focus on the psychological underpinnings of individual traffic behaviors.

\section{Causal and statistical assumptions of latent variable models and network 84 models}

85 When discussing latent variable models, a distinction is often drawn between measurement 86 models and structural models ${ }^{69}$. Measurement models describe the relationships between latent 87 variables (e.g. errors) and observed variables (e.g. miss observing a pedestrian, misestimate the 88 speed of another vehicle). Structural models concern interrelationships between latent variables 89 (and observed variables assumed to be measured without error). Now, as the name suggests, in a 
90

91

92

93

94

95

96

97

98

99

100

101

102

103

104

105

106

107

108

109

110

111

112

113

114

115

116

117

118

119

120

121

122

123

124

125

126

127

measurement model, the relationship between the latent and observed variables is understood as one of measurement. This idea is most readily compatible with giving the latent variables a realist interpretation, i.e. understanding them as existing independently of the act of measurement ${ }^{15}$. Even though the matter is rarely explicitly discussed in traffic psychology, the realist interpretation seems in line with how latent variables are used in practice: separate psychological processes are taken to underlie the latent variables (errors and violations), and causal language is used when describing the relationships between the latent and observed variables $^{2,3,4}$. Under the realist interpretation, the relationship between the latent variable (the measured quantity) and observed variables is seen as one of causation: the measured quantity is seen as causing variation in the observed variables similarly to how temperature causes variation in thermometers ${ }^{70}$. It is naturally possible to interpret latent variables in a non-realist fashion, e.g. as mere statistical summaries, in which case the relationship can be understood as a logical one instead of a causal one ${ }^{15}$. However, if one is committed to ideas such as estimating the values of parameters, evaluating the position of a subject on the latent variable or assessing the truth of a claim involving a latent variable, it appears that latent variables need to be given a realist interpretation ${ }^{15}$.

The realist interpretation of latent variables is naturally compatible with viewing variation in the observed variables as being due to either the latent variables or measurement error, and allowing no covariance among the observed variables once these effects are accounted for ${ }^{15}$. Such relationships are schematically depicted in Figure 1. Attributing causal power to latent variables in this manner renders the observed variables inert, interchangeable reflections of the latent properties being measured ${ }^{15,70}$. The idea that observed variables are rendered statistically independent when conditioning on the latent variable(s) is known as the assumption of local independence ${ }^{15}$ and it is problematic in at least two cases: 1) when several latent variables prove to influence a given set of observed variables (lack of unidimensionality) and 2) when direct causal connections exist among the observed variables.

The problematic nature of the unidimensionality assumption is concretely reflected in the common practice of representing errors and violations as unweighted or weighted ${ }^{16}$ sum scores. The following example is related to the unidimensionality (or the lack thereof) of items related to the subscale of violations in the DBQ. Consider two imaginary persons filling in the DBQ: John, known for his quick temper, answers the three items related to aggressive behavior with the option "nearly all the time", and reports performing no other violations, thus obtaining the sum score of 21. Bill, on the other hand, known for his careful nature, chooses the option "never" to the aggression-related items and the option "hardly ever" or "occasionally" to the other violation items. As there are many more items related to non-aggressive violations than to aggressive ones, both respondents receive identical scores, even though their behavioral profiles are quite different. It is naturally possible to react to this challenge within the latent variable framework by creating a more complex model. For instance, "ordinary violations" and "aggressive violations" 
128 can be modelled as separate latent variables ${ }^{5}$. Still, the unidimensionality of the items related to

129 these further latent variables remains an open research question.

130 The latter idea, the possibility of direct causal connections among observed variables is less often

131 discussed, and it motivates applying network models in the first place. For instance, driving

132 under the influence of alcohol or driving fast may predispose the driver to other violations and

133 errors, and the consequences of these two violations are likely to be different. Assuming the

134 existence of such direct associations between observed variables is in fact quite a drastic move,

135 since it necessitates viewing the relationship between the latent and observed variables as

136 something else than measurement, perhaps as one of constitution ${ }^{71}$. The metaphor of interacting

137 birds forming a flock has been used to describe the nature of the relationship ${ }^{71}$.

138 Finally, previous research has indicated equivocal factorial structures for the DBQ. This is 139 evidenced by

1) Different factor structures being obtained for the same version of the instrument. For instance, the often-used 27 -item questionnaire is thought to reflect two ${ }^{17,18}$, three ${ }^{19}$ or four ${ }^{20}$ different psychological processes, with exploratory analyses sometimes indicating even more latent variables ${ }^{21}$;

2) Typically high cross-loadings of items on factors ${ }^{5,22}$;

3) Complex factor structures needed to adequately fit the data, either by specifying second-order factors ${ }^{3}$ or a general factor ${ }^{20}$ and

4) Failures of the test of measurement equivalence across certain subgroups $s^{4,5,22}$.

Observations such as these function as an empirical motivation for creating the network models reported in the present paper.

150 Figure 1 shows schematically the fundamental assumptions of latent variable models. Individual behaviors, such as speeding or misjudging speed, are assumed to reflect the level of the underlying latent variable and measurement error. Background factors such as enjoying speed figure as predictors of the latent variables. Importantly, background factors are unrelated to individual driver behaviors, which are assumed causally inefficacious. Typically, a dependent variable of interest, such as the number of crashes, is regressed on sum variables representing violations and errors ${ }^{16}$. One distinct benefit of such models is their simplicity: if the latent variables indeed manage to capture the important commonalities among the individual behaviors, they provide an extremely parsimonious representation of the data. In addition, the model shown in Figure 1 is, on purpose, a rather simple example of a structural equation model; these models are a flexible tool that would allow the researcher to add more latent variables and to represent much more complex relationships among them. Further, the model shown in Figure 1 is not intended to summarize theoretically or practically important findings in traffic research, but rather, together with Figure 2, to illustrate the kinds of relationships that may obtain between the 
Network models, such as the hypothetical model shown in Figure 2, are naturally suited to investigating direct associations among traffic behaviors. Individual behaviors are also assumed to be directly affected by background factors. Further, two background variables, stress and congestion, that were not included in Figure 1, are shown in Figure 2. This illustrates the possibility that there may exist background factors that are related to only one of the observed variables. Such relationships can be more naturally accommodated in network models than in latent variable models. In network models, the absence of an edge between two nodes is interpreted as showing that when the effects of all other nodes in the network are controlled, the two nodes are rendered statistically independent ${ }^{23}$. The non-zero edges can be interpreted as potential causal connections ${ }^{23}$, logical relationships of entailment ${ }^{24}$, reflections of the influence of an unmodelled latent variable affecting both nodes ${ }^{66}$, conditioning on a collider (a common effect of both nodes $)^{67}$ or perhaps semantic relationships of the nodes being close to each other in meaning. Finally, a cluster of nodes may reflect the effect of a latent variable influencing all the nodes in the cluster ${ }^{25}$.

Network models are valuable tools in exploratory analysis of the data: they enable representing pairwise associations among variables quickly, intuitively and efficiently. However, network models assume that the relationships among the observed variables (in this case, the driver behaviors) are not due to latent variables at all, which is a remarkably strong assumption. In this sense, latent variable models and network models can be seen as having complementary strengths and weaknesses ${ }^{65}$.

This paper conceptualizes the behavior of newly licensed drivers as an interacting network of component behaviors. First, the dynamics of the relationships between the typical levels of individual violations and errors during the first three years post-licensure are represented as the between-person network ${ }^{26}$, which enables assessing direct associations between behaviors and judging the centrality of the behaviors in the network. Second, relationships between driver behaviors and background factors are assessed in a cross-sectional analysis using data collected at six months post-licensure. Importantly, the background factors are directly linked to individual driver behaviors rather than latent variables or sum scores. Third, individual driver behaviors recorded at 6 months post-licensure are used for predicting crashes occurring during the following $2 \frac{1}{2}$ years. Different predictive models are compared by building the models in one subset of data and testing them in another one. 
199 Materials and Methods

\section{Data and participants}

201 This study is based on the archival dataset collected in the longitudinal Cohort II study in 2001 2022005 on new and novice drivers in the $\mathrm{UK}^{27}$. The initial sample size was 20,512 with four waves 203 of data collection taking place after the respondents had passed their driving tests. The numbers 204 of responses and response rates were as follows: 10,064 at 6 months (49\%), 7,450 at 12 months 205 (36\%), 4,189 at 24 months ( $26 \%$ ) and 2,765 at 36 months (26\%). The age distribution of the

206 respondents corresponded to that of the population of newly licensed drivers, with $59 \%$ of

207 respondents under the age of 20 at the first wave of data collection and $76 \%$ under the age of 25 .

208 Women, on the other hand, were overrepresented: at the first wave, $64 \%$ of the respondents

209 were females. The dataset has been previously used in several studies ${ }^{16,18,20,28}$ and is freely

210 available online ${ }^{29}$.

211 The cross-sectional network analyses were performed on data obtained at six months post-

212 licensure. Only cases with non-missing data in all variables were included, resulting in a sample

213 size of 8858 . The respondents included in this analysis had a mean age of 22.51 years $(\mathrm{SD}=$

214 7.95), and $64 \%$ of them were female. Due to the drop-out described above, a much smaller

215 number of cases was available for forming the between-person network: the model was built

216 based on 1173 cases with no missing data. The between-person network was formed by

217 averaging responses across the four time points. The respondents in the between-person analysis

218 had a mean age of 24.04 years $(\mathrm{SD}=9.62)$, and $71 \%$ of them were female. The regression

219 analyses were based on cases with no missing values on any of the predictor variables or the

220 number of crashes variable, resulting in $\mathrm{N}=1152,69 \%$ female.

\section{Measures}

222 Data collected using the Driving Experience Questionnaire (DEQ) that includes a 39-item

223 version of the DBQ was used. In addition to the DBQ items, items referred to as "background

224 factors" were used. The responses to the DBQ items were recorded on a six-point Likert scale

225 ("never" to "nearly all the time"), and were intended to assess the two constructs of violations

226 and errors. Example items include How often do you disregard the speed limit on a motorway

227 and How often do you select the wrong gear when wanting to go into reverse, respectively. The

228 response options for the background factors were as follows. Drivers' self-image was assessed

229 on a seven-point scale anchored to the end points of a continuum (e.g., "inattentive" to

230 "attentive"). The self-perceived improvement needs were recorded on a three-point scale (e.g.,

231 "no improvement needed in controlling the car" to "a lot of improvement needed in controlling

232 the car"). Attitudes were assessed on a five-point scale ranging from "Strongly disagree" to

233 "Strongly agree"; an example item reads Decreasing the motorway speed limit is a good idea. In

234 addition, self-reported age, sex and mileage at six months post-licensure were used as predictors

235 of public road crashes (both major and minor crashes) in the Poisson regression analyses. 


\section{Procedure}

237 The "Learning to drive questionnaire", which includes the question items related to attitudes,

238 was filled in after the practical driving test, prior to responding to the DEQ. Informed consent

239 was inferred from returned postal questionnaires in accordance with the social research

240 guidelines of Department of Transport.

\section{Statistical analyses}

242 Network analyses

243 The network analyses were based on the common, widely used 27-item version of the $\mathrm{DBQ}^{3,5,30}$

244 together with items related to driving under the influence of alcohol and drugs, using the cell

245 phone while driving and having to brake or swerve to avoid an accident, resulting in 31 DBQ

246 items. These latter items are often omitted from latent variable models of the DBQ because of

247 their low factor loadings ${ }^{5}$, but they were now included due to their potential significance as

248 determinants of other driving behaviors. Two types of network models were estimated. The

249 cross-sectional network model was based on the 31 DBQ items together with nine items related

250 to the background factors. The latter were chosen from among candidate variables using a series

251 of exploratory network analyses (Supplementary Figs. S1-S3). The between-person network ${ }^{26,31}$

252 was formed by calculating average scores for each respondent across the four time points to

253 represent the overall pattern of driving behaviors during the first years of learning to drive. The

254 benefit of between-person networks is that they are less susceptible than cross-sectional networks

255 to spurious effects and reporting biases ${ }^{26}$ such as mood-congruent recall ${ }^{32}$, as different biasing

256 effects are likely to cancel each other out.

257 In both models, individual questionnaire items correspond to the nodes of the network, while the

258 edges represent partial correlations controlling for all other nodes. The procedure attempts to

259 uncover a graph known as a Gaussian Graphical Model in which each node is independent of the

260 rest given the values of immediately neighboring nodes as described by the Markov properties ${ }^{33}$.

261 In other words, an edge connecting two nodes indicates their conditional dependence given the

262 other nodes. The edge weights were scaled to the joint maximum value of the two models to

263 ensure the comparability of the results. All network analyses were performed in $\mathrm{R}^{34}$ using the

264 packages qgraph $^{35}$ and bootnet ${ }^{36}$.

265 Some of the partial correlations are likely to differ from zero because of sampling variation and

266 can be thought of as false positive findings ${ }^{9}$. For this reason, the graphical LASSO ${ }^{37}$ was used in

267 estimating the networks based on polychoric correlations. The procedure constrains low values

268 of partial correlations to zero, thus resulting in sparse models ${ }^{23}$. The level of sparsity is

269 determined by the tuning parameter $\lambda$, the value of which was chosen based on the Extended

270 Bayesian Information Criterion (EBIC), as this has been shown to work well in retrieving the

271 true network structure ${ }^{38}$, especially if the true model is sparse ${ }^{23}$. The hyperparameter $\gamma$ used in

272 EBIC model selection was set to the recommended default value of $0.5^{38}$. The locations of the

273 nodes were determined using a modified version of the Fruchterman-Reingold algorithm ${ }^{39}$ for 
274 weighted networks ${ }^{35}$, which places strongly connected nodes that have many edges in common

275 close to one another.

276 The importance of the individual nodes in the network was assessed by calculating three indices

277 of centrality: strength, betweenness and closeness. Strength refers to the sum of edge weights of

278 the focal node, closeness to the reciprocal of the sum of distances from the focal node to all other

279 nodes and betweenness to the number of shortest paths between two nodes that pass through the

280 focal node. The values of the centrality indices were standardized to ensure comparability

281 between networks and between studies. Further, the generalization of Zhang's local clustering

282 coefficient ${ }^{40}$ to signed networks ${ }^{41}$ was calculated to represent the redundancy of the focal node.

283 This coefficient was used due to its sensitivity to weak edges ${ }^{42}$. The stability of the centrality

284 indices and the accuracy of edge weight estimates were assessed by bootstrap analyses

285 (Supplementary Figs. 4-5) using the bootnet package ${ }^{36}$. Further, the stability of the indices was

286 quantified by the correlation stability (CS) coefficient, the value of which should preferentially

287 exceed $0.5^{36}$ (the values are reported in Supplementary Table S5).

288 Regression analyses

289 The 39 DBQ variables, together with the variables mileage, age and sex, recorded at six months

290 post-licensure, were used as predictors of the number of crashes during the latter waves of data

291

292

293

294

295

296

297

298

299

300

301

302

303

304

305

306

307 collection in a Poisson regression model with a logarithmic link function. . The regression analyses were based on all $39 \mathrm{DBQ}$ items to maximize the predictive power of the model. The analysis was based on the idea of maximizing predictive accuracy via minimizing generalized cross-validation error (GCVE, operationalized as the deviation score), as described by Chapman et al. ${ }^{43}$. The minimization of the GCVE aims at creating models that explain the maximum amount of variance without overfitting the model to data at hand. This is achieved by trading some increase in bias to a reduction in variance ${ }^{43}$.

The data set was randomly split into a training set and a test set $\left(75 / 25\right.$ ratio, with $\mathrm{N}_{\text {training }}=864$ and $\mathrm{N}_{\text {testing }}=288$ ). The uneven ratio was chosen to enable a sufficiently large number of crossvalidation splits, with initial model fitting and cross-validation taking place within the training set, followed by fitting the same model in the test set using the R-package glmnet ${ }^{44}$. The same penalty, controlled by the regularization hyperparameter $\lambda$, was applied to all the predictors, which were standardized prior to analysis. Self-reported mileage was also log-transformed.

Three Poisson regression models were fit to the training and test data. First, an elastic net mode ${ }^{45}$, was formed based on a grid search of optimal values of the hyperparameters $\alpha$ (elastic net mixing parameter) and $\lambda$ (regularization parameter), the values of which were chosen by minimizing the value of GCVE in a 10 -fold cross-validation analysis. The elastic net combines penalties based on squared sums of regression coefficients (ridge) and the sum of their absolute

309 values (lasso); it thus performs variable selection similarly to the lasso, and performs well with

310 correlated predictors similarly to ridge regression ${ }^{45}$. Second, a ridge regression model

311 (regularization without variable selection) was obtained by taking the cross-validated value of $\lambda$

312 (regularization parameter) and setting the value of $\alpha$ (elastic net mixing parameter) to zero.

313 Finally, the naïve Poisson model with all predictors and no regularization was fitted to the data. 
314 Model fit was assessed in the test data set $(\mathrm{N}=288)$ using several descriptive statistics. First, 315 residual variance was quantified using deviance residuals ${ }^{44}$ and mean squared error; second, the

316 similarity of the predicted values and the actual values were assessed by calculating their Pearson

317 correlation; third, McFadden's Pseudo $\mathrm{R}^{2}{ }^{46}$ was used for assessing how much model fit

318 improved from the null model; finally, the min-max index was calculated as MinMax =

$319 \sum_{i}\left(\frac{\min \left(y_{i} \hat{y}_{i}\right)}{\max \left(y_{i}, \hat{y}_{i}\right)}\right) / n$.

320 Data availability

321 The datasets analysed during the current study are available in the UK Data Service data archive

322 at https://discover.ukdataservice.ac.uk/doi?sn=5985\#2.

323

324 Results

325 Network analyses

326 The results reported below are based on the maximum number of cases available for the

327 respective analyses as described in the Methods section $(\mathrm{N}=1173$ for the between-person model

328 and $\mathrm{N}=8858$ for the cross-sectional network model, respectively). First, the between-person

329 network is shown in Fig. 3. It illustrates between-person differences in the connectivity of the

330 driver behaviors during the first three years of learning to drive. Errors are shown as striped blue

331 nodes and violations as striped red nodes. The presence of an edge between two nodes represents

332 their conditional dependence when controlling for all other nodes. 
333 First, in outline, violations and errors occupy different regions of the graph, and their distinction

334

335

336

337

338

339

340

341

342

343

344

345

346

347

348

349

350

351

352

353

354

355

356

357

358

359

360

361

362

363

364

365

366

367

368

369

370

371 seems a sensible rough description of the data. However, if all violations and all errors were reflections of respective latent variables, we would expect all violations and all errors to be interconnected ${ }^{23}$ and for all violations and errors to be independent. In contrast, thematically related violations are clustered together and connected by strong edges, aggression-related nodes ( $22, \mathrm{v} 4, \mathrm{v} 9)$ and speeding-related nodes ( $55, \mathrm{v} 11)$ being a case in point. The edges are interpreted as showing, e.g., that drivers who were more likely than others to exceed speed limits within residential areas (v5) were also more likely to do so on highways (v11). Similarly, nodes related to substance abuse $(\mathrm{v} 8, \mathrm{v} 12)$ were connected to each other and few other behaviors, except using the cell phone while driving (v6). Similar considerations apply to errors. For instance, the nodes related to forgetting something (e3, e6) shared a strong edge and were connected to few other nodes except absent-mindedness (v13).

Second, certain violations and errors were connected by relatively strong edges. Notably, drivers who exceeded speed limits within residential areas (v5) were more likely than others to tailgate other drivers (e15). Further, the tailgating drivers were more likely to need to brake or swerve to avoid an accident (e19). Similarly, crossing junctions against a red light (v3, a violation) was connected by roughly equally strong edges to other violations and errors.

Third, not all thematically related nodes were connected by strong edges, the two items related to perceiving traffic signs (e8, e16) being a case in point. Still, both shared strong edges with other errors; for instance, misreading signs (e8) with getting into a wrong lane (e14). Finally, a methodological note is in order: differences between edge strengths can be interpreted only if their confidence intervals are not excessively wide. Bootstrap analyses assessing this are reported in Supplementary Figs 4-5).

\section{---------- Insert Figure 3 here -----------}

Interpreting large networks such as those in Fig. 3 becomes easier with examining indices of node centrality and clustering. The interpretability of the indices themselves depends on their stability, which can be judged by calculating the correlation stability coefficient based on bootstrap tests (Supplementary Table S5). The indices whose CS-coefficient exceeded the recommended value of $0.5^{36}$ are shown in Fig. 4.

Both strength centrality (associations with immediate neighbors) and closeness centrality (associations with all nodes) of the between-person network indicated the presence of a group of nodes that were especially central in the network. Closeness centrality is perhaps the more revealing in this context, as it capitalizes less on single strong edges. The node with the highest closeness centrality was v5 (speeding within a residential area), followed by nodes v3 (crossing junction on red), e15 (tailgating), e10 (queuing, nearly hit car), e17 (fail to check mirror) and v11 (speeding on motorway); nodes e10, v5, e15 and v3 had also high strength centralities. In addition, nodes e14 (getting into a wrong lane) and e7 (failing to notice pedestrians) had a high 
372 strength centrality. In general, the nodes along the path connecting speeding with various errors

373 (v11-v5-v3-e7-e17-e15-e10 or v11-v5-e15 etc.) were central. Figure 4 also shows Zhang's

374 clustering coefficient, according to which certain nodes, most notably v4 (become angered, give

375 chase) and node e3 (forget where left car) contained little unique information.

376

377 Insert Figure 4 here

378

The cross-sectional network model (Fig. 5) shows the DBQ variables in relation to various

380 background factors: attitudes (yellow), self-judged improvement needs (green) and self-image as

381 a driver (lilac). The model is based on data collected at the first time point, 6 months postlicensure. It appears at the first sight quite different from the between-person model, but this is largely because the node placements are different due the use of the Fruchterman-Reingold algorithm. Similarities between the two models are revealed by examining the connection strengths and the centrality indices (Fig. 6). The indices whose CS-coefficient exceeded the recommended value of $0.5^{36}$ are shown in Fig. 6 . The speeding-related nodes (v5 and v11) were again central together with node e7. Node e16 (missing give way signs) now had a high strength centrality, while in the between-person network the corresponding centrality value was average; on the other hand, the connectivity patterns of the node were similar. The path v11-v5-e15 was present also in this model, even though node e 15 was now slightly less central. A prominent difference between the models was that the aggression-related nodes $\mathrm{v} 2, \mathrm{v} 4$ and $\mathrm{v} 9$ were more central in the cross-sectional model. Insert Figure 5 here

Looking at the background factors, the self-judged improvement needs were strongly interconnected. They were, however, also related to driving behaviors in a revealing pattern. In general, they shared negative associations with different violations. In particular, the judged need for improvement in changing lanes (in4) was related to less speeding (v11) and less pushing into a lane (v7), while the perceived need of improving controlling the car (in1) was associated with having problems with gears (e1) and car controls (e4). Negative attitudes to speeding (a1) and overtaking on the inside (a2) were related to fewer self-reported behaviors of those kinds (v11 and $\mathrm{v} 1$, respectively). On the other hand, perceiving oneself as a fast driver (si2) was positively associated with speeding ( 55 and $\mathrm{v} 11$ ) and racing from the lights (v10). The drivers' selfjudgment of themselves as irritable (si1) was closely associated with the anger-related nodes, even though the node was quite redundant as judged by the clustering coefficient. Self-perceived need of improvement in hazard-perception (in3) had a similarly high value of the clustering 408 coefficient. 


\section{Regression analysis}

413

414

415

416

417

418

419

420

421

422

423

424

425

426

427

428

429

430

431

432

433

434

435

436

437

438

439

440

441

442

443

444
The self-reported driving behaviors, age, sex and mileage at the first time point were used in predicting subsequent crashes. As seen in Table 1, the naive Poisson model fit the training data best (as it should), but its performance deteriorated notably in the hold-out data. In fact, the model fit the hold-out data worse than the null model without predictors, offering a dramatic demonstration of how such models sometimes overfit data. The elastic net model fit the training data the worst, but its fit to the hold-out data was almost identical to that of the ridge regression model and is to be preferred due to its parsimony (16 vs. 42 parameters, respectively).

-Insert Table 1 here

Table 2 shows the regression weights of the elastic net model that are low, partly because of the dual penalization involved. The driving behaviors included in the model comprise various violations and errors. Variables related to failing to notice pedestrians, problems with car controls and different forms of aggressive behavior had the highest regression weights. Hitting something when reversing, which is itself a minor crash, also predicted subsequent crashes.

Insert Table 2 here

\section{Discussion}

The present paper put forward three main ideas: 1) Individual violations and errors correlate because direct, possibly causal, relationships exist between perceptions, thoughts, emotions and actions in traffic. In particular, individual violations and errors were not assumed to reflect the effects of underlying, in principle unobservable variables, such as violation-proneness and errorproneness. 2) Hypotheses concerning these relationships can be formulated in a data-driven manner by estimating the strengths of associations between pairs of variables while controlling for all other variables in a network model. Similar ideas of network models as hypothesisgenerating structures have been expressed in the context of psychopathology research ${ }^{8,47} .3$ ) Background factors such as attitudes, self-image etc. are directly related to individual traffic behaviors such as speeding or drunk driving; similarly, individual traffic behaviors are related to crash risk, and these relationships can be explored by developing criterion-keyed psychometric scales $^{43}$. Finally, to understand different errors and violations, one needs to consider the context in which they take place rather than assuming that they reflect stable traits of the individuals 
445 (error-proneness and violation-proneness). The empirical part of the study dealt with the first 446 three questions. Research related to the fourth is also discussed, as the context-sensitivity of 447 behavior is an important motivation for network models of human behavior.

448 The dynamics of the relationships between the typical levels of individual violations and errors 449 were examined by constructing the between-subject network. The analysis showed that drivers 450 who were more likely than others to exceed speed limits within residential areas were also more 451 likely to tailgate the vehicle in front; further, the tailgating drivers were more likely than others 452 to need to brake or swerve to avoid an accident. These associations were interpreted as causal 453 hypotheses: drivers who exceed speed limits will likely catch up with the traffic flow and may as 454 a consequence need to react. Similarly, crossing a junction against a red light (a violation) was associated with failing to notice pedestrians and with other errors. Importantly, such associations between violations and errors are difficult to accommodate within latent variable models of driver behavior.

Exceeding speed limits within residential areas appeared as the most closeness central and the second-most strength central node in the between-subject network. Insofar as the centrality of a node can be taken to reflect the causal connections emanating from that node, a successful intervention of reducing speeding might affect the other behaviors directly or indirectly linked to it. Care must be taken with such interpretations, since the edges in the network may well reflect other factors than a cause-effect relationship ${ }^{23,24,25,66,67}$, but at the very least, such causal hypotheses can be formulated based on the present results. In fact, network models benefit traffic research precisely in that they encourage thinking of the dynamics of the behaviors. In contrast, latent variable models remain silent in this respect, as they conceptualize individual behaviors as causally passive indicators of the latent variables ${ }^{15}$. Thus, if we take the latent variable view seriously, we can only influence individual behaviors through manipulating the latent variables: whether we want to reduce drunk driving or speeding, we should aim at the drivers' rulebreaking tendencies, because influencing an individual violation has no effect on other behaviors under the latent variable view.

472 The relationships between background factors and traffic behaviors were examined in the crosssectional network model based on data collected six months post-licensure. Drivers who perceived themselves in need of improving lane-changing skills were less likely to report speeding and pushing into a lane. Further, the respondents reported behaving according to their attitudes: a preference of decreasing speed limits was associated with less speeding. On the other hand, perceiving oneself as a "fast driver" was associated with more speeding and racing from the lights. In short, individual background factors were related to driver behaviors in an understandable pattern.

480 Even though it is difficult to explain direct associations between driver behaviors using latent variable models, they pose a challenge to the network view: certain behaviors are more likely to occur together than others; why is this so if there are no latent variables? One can begin to answer by considering relationships between behavior and environment, as illustrated by the following quote from a study on driver irritation and aggression: 
485

486

487

488

489

490

491

492

493

494

495

496

497

498

499

500

501

502

503

504

505

506

507

508

509

510

511

512

513

514

515

516

517

518

519

520

521

522

523

524

525

"... Drivers who enjoy a somewhat faster speed than other drivers will more often be obstructed by other traffic, and therefore they will become irritated more often and be more likely to educate other road users. They probably also will become more irritated than other drivers when obstructed, because they want a faster progress" 55 .

In other words, people have characteristics such as emotions, attitudes and personality components $^{56}$ that affect their behavior in traffic, which is not only something to be explained, but also a variable that feeds back into the system of emotions, perceptions and other behaviors. Further, not all behavioral characteristics are equally compatible with each other. For instance, the people described in the quote may be unlikely to make errors related to car controls, which are perhaps related to lack of experience or lack of interest in cars. In technical terms, network models exhibit non-trivial topology ${ }^{47}$. Further, they can - as also demonstrated in the present study - accommodate background variables such as beliefs, and account for why beliefs, feelings and behaviors become aligned ${ }^{10}$. For instance, the drivers described in the quote are perhaps likely to consider speed limits in general too low to avoid cognitive dissonance between their actions and beliefs.

The above example illustrated stable differences between people. However, people do not always behave in a stable manner; rather, their behavior is context-dependent. The idea has much in common with the cognitive-affective personality system ${ }^{57}$ (CAPS) theory developed within personality psychology, which posits if-then rules that describe how someone typically reacts in a certain type of a situation. CAPS is influenced by connectionist models, and characterizes behaviors, memories and emotions as differently activated by the situational context and each other. The interconnected elements are described as a network in which activation is sustained by feedback loops. Such situation-specific rules might apply to, e.g., young people driving with their friends vs. with a mother and a baby on board. In the former situation, the driver's repertoire of certain risky behaviors is more highly activated and activation spreads through excitatory links. For example, the threshold of speeding might be lowered, activating an impulse to race other drivers. In short, the network of driving behaviors can be seen as being in two qualitatively different states distinguished by the connection strengths between the behaviors. As another example, it has been shown that young mothers have an elevated crash risk when driving with an infant passenger compared to driving alone ${ }^{58}$; the dynamics of their behavior in these contexts are likely to differ.

In addition to presenting network models of driver behavior, this study involved predicting crashes from individual behaviors. In contrast, previous self-report studies have mainly used latent variables in crash prediction (even though see Wallén-Warner et al. ${ }^{59}$ for an exception). The novel contributions of the present study were three-fold. First, crashes were truly predicted from earlier behavior. Second, predictive models were first fit in a training subsample and then verified in an independent subsample of data. Third, regularized regression was used to prevent overfitting.

Three predictive models were tested, out of which the elastic net model ${ }^{45}$ and the ridge regression model fit the hold-out data roughly equally well, and notably better than the naïve Poisson regression model. The elastic net model has the benefit of being more parsimonious than 
526 the ridge regression model, so it is to be preferred, other things equal. In the elastic net model, 527 predictors included displaying anger while driving and driving fast (disregarding speed limits 528 and racing from traffic lights). Further, errors in visual search (failing to notice people, missing 529 signs) and in controlling the car (driving off at wrong gear, forgetting the handbrake) were 530 included in the model. Interestingly, hitting something when reversing, which is itself a minor $531 \mathrm{crash}^{16}$, predicted future crashes. Remarkably, the naïve Poisson model including all predictors 532 fit the hold-out subsample worse than the null model with no predictors, offering a dramatic 533 demonstration of the dangers of overfitting.

534 The present study has its limitations. First, network models are motivated by modelling the 535 components of a phenomenon, with a component defined as something having unique causal relations with the rest of the network ${ }^{56}$. The DBQ has been psychometrically developed to maximize reliability, which has resulted in a certain redundancy of the items. In this study, this shows as high values of the clustering coefficient for the nodes related to aggression and speeding, which could in future studies be represented by single nodes. On the other hand, developing a novel questionnaire describing potentially causally related behaviors, thoughts and emotional reactions is another option. Further, the behaviors examined here are influenced by other road users, which could not be accounted for. For instance, aggressive behavior is difficult to understand without knowing something about its target. It is naturally possible to take an even more critical perspective and argue that none of the relationships indicate potential causal relationships and driver behaviors should continue to be viewed as being caused by latent variables. Even so, the present study presents a challenge: why are variables that commonly load on different factors strongly correlated? Finally, it is likely that general psychological characteristics, such as impulsivity, conscientiousness, neuroticism, agreeableness, attention and memory capacity etc. would explain the associations observed in the present study. The fact that information on such characteristics was not available is a clear limitation of the present study. In addition to the substantive questions, some methodological decisions were problematic. First, polychoric correlations that estimate normally distributed variables underlying ordinal input variables were used, even though the variables were positively skewed. Although the practice is widespread in psychometric network models, it is not optimal ${ }^{60}$. Further, listwise deletion of missing data was performed; less wasteful methods are under development for network models but not yet available ${ }^{60}$. Further, it has been shown that young respondents may give exaggerated responses to questions they find funny, a response bias dubbed "mischievous responding" 61 ; this could affect items such as drug use while driving. On the other hand, if such biases are transient in nature, between-person networks are likely a suitable method to use ${ }^{26}$.

560 Choosing the correct components of driver behavior is a central issue to be tackled in future 561 network models of driver behavior. In addition, future studies should aim at developing a 562 network theory of driver behavior instead of merely applying a novel modelling tool. A recently developed intricate error taxonomy ${ }^{54}$ might provide a good starting point together with factors contributing to such errors. Also, the need for self-report studies will remain in the future even though studies involving instrumented vehicles have become ever more intricate ${ }^{62}$, because 
566 physical measurements are underdetermined psychologically ${ }^{29}$ : for instance, a sudden

567 acceleration can be due to either racing from the lights or unfamiliarity with car controls.

568 Further, the context-dependent nature of driving needs to be taken into account in future studies.

569 Drivers may behave differently depending on who they are traveling with; similarly,

570 investigating drivers' developing situation-awareness of when to desist from violating rules has

571 been called for ${ }^{29}$, and network models are an ideal tool for investigating such developmental

572 trajectories $^{31}$. Another fascinating future direction is to consider the effects of the driver's state:

573 being fatigued, intoxicated, stressed, in a hurry or in a strong emotional state could conceivably

574 cause the network of driver behaviors to occupy qualitatively different states. Existing task

575 analyses and models of driving situations ${ }^{63,64}$ are likely to be a good starting point, because

576 individuals may behave in a more-or-less stable manner in a certain type of a situation, but not

577 across situations ${ }^{57}$. Intriguing avenues for future research await those willing to look into the

578 networks of driver behavior.

\section{Conclusions}

580 Representing violations and errors on the road as interconnected networks of behaviors,

581 cognitions and emotions makes it possible to formulate data-driven hypotheses on causal

582 connections between individual violations and errors. For instance, exceeding speed limits may

583 make it more likely for drivers to end up tailgating other vehicles, which in turn may make it

584 more likely that they need to brake or swerve to avoid accidents. In contrast, previous

585 psychometric work has been based on the latent variable view, according to which individual

586 errors and violations function as (nothing but) reflections of underlying psychological properties,

587 error-proneness and violation-proneness. It is argued herein that this is an overly simplified view

588 of the determinants of traffic behavior, and that the network view provides a useful novel point

589 of view in this respect.

590 More generally, network models show promise for bridging a gap between experimental and

591 theoretical work in traffic research on the one hand and self-report-based research on the other

592 hand. The latter has commonly assumed the existence of a small number of mutually exclusive

593 psychological mechanisms whose operation is reflected in respective sets of driver behaviors

594 (e.g. violations and errors) that can be represented using latent variables or sum scores. On the

595 other hand, the importance of individual driver behaviors, such as speeding, is recognized in

596 theories of driver motivation ${ }^{48,49}$, studies that aim at determining reasons for speeding ${ }^{50-52}$ and

597 engineering models of accidents ${ }^{53}$. Similarly, an error taxonomy involving action errors,

598 cognitive and decision-making errors, observation errors and information retrieval errors has

599 been proposed ${ }^{54}$, indicating the need to differentiate errors in a fine manner. Network models that

600 focus on individual driving behaviors and their interrelationships offer a novel point of view

601 from which to integrate the results of self-report studies with these lines of research.

602 In addition to presenting network models of driver behavior, this paper involved predicting

603 crashes based on individual errors and violations. This was done using cross-validated penalized

604 regression analysis, which resulted in a model that was both predictive of accidents and

605 generalizable to new data. Similarly to the network models, the predictive models can be 
606 contrasted to those used in prior psychometric traffic research, in which latent variables have 607 been used as predictors of crashes: the current paper argues that it is important to consider the 608 role of individual traffic behaviors in determining crashes and to build predictive models from 609 this point of departure.

\section{Acknowledgments}

611 I would like to thank Jami Pekkanen and Otto Lappi for constructive feedback on earlier versions

612 of the manuscript and Jami Pekkanen for helpful discussions on performing the regression

613 analyses.

\section{References}

615 1. Reason, J., Manstead, A., Stradling, S., Baxter, J. \& Campbell, K. Errors and violations on the 616 roads: a real distinction? Ergonomics 33, 1315-1332 (1990).

617 2. Mesken, J., Lajunen, T. \& Summala, H. Interpersonal violations, speeding violations and their 618 relation to accident involvement in Finland. Ergonomics 45, 469-483 (2002).

619 3. Lajunen, T., Parker, D. \& Summala, H. The Manchester Driver Behaviour Questionnaire: A 620 cross-cultural study. Accid. Anal. Prev. 36, 231-238 (2004).

621 4. Stephens, A. \& Fitzharris, M. Validation of the Driver Behaviour Questionnaire in a 622 representative sample of drivers in Australia. Accid. Anal. Prev. 86, 186-198 (2016).

623 5. Mattsson, M. Investigating the factorial invariance of the 28-item DBQ across genders and age 624 groups: An Exploratory Structural Equation Modeling Study. Accid. Anal. Prev. 48, 379-396 625 (2012).

626 6. Koppel, S., Stephens, A., Charlton, J., Di Stefano, M., Darzins, P., Odell, M. \& Marshall, S. 627 The Driver Behaviour Questionnaire for older drivers: Do errors, violations and lapses change 628 over time? Accid. Anal. Prev. 113, 171-178 (2018).

629 7. Borsboom, D. \& Cramer, A. Network analysis: an integrative approach to the structure of 630 psychopathology. Annu. Rev. Clin. Psychol. 9, 91-121 (2013).

631 8. Fried, E. \& Cramer, A. Moving forward: challenges and directions for psychopathological 632 network theory and methodology. Perspect. Psychol. Sci. 12, 999-1020 (2017).

633 9. Costantini, G., Epskamp, S., Borsboom, D., Perugini, M., Mõttus, R, Waldorp, L. \& Cramer, 634 A. State of the aRt personality research: A tutorial on network analysis of personality data in R.

635 J. Res. Pers. 54, 13-29 (2015).

636 10. Dalege, J., Borsboom, D., van Harreveld, F., van den Berg, H., Conner, M. \& van der Maas,

637 H. Toward a formalized account of attitudes: The Causal Attitude Network (CAN) model.

638 Psychol. Rev. 123, 2 (2016).

639 11. Van Der Maas, H., Dolan, C., Grasman, R., Wicherts, J., Huizenga, H. \& Raijmakers, M. A 640 dynamical model of general intelligence: the positive manifold of intelligence by mutualism.

641 Psychol. Rev. 113, 842-861 (2006).

642 12. Lappi, O. \& Rusanen, A. Turing machines and causal mechanisms in cognitive science.

643 Causality in the sciences, 224-239 (2011).

644 13. Bechtel, W. Mechanisms in Cognitive Psychology: What Are the Operations? Philosophy of 645 Science 75, 983-994 (2008).

646 14. Boag, S. Explanation in personality psychology: "Verbal magic" and the five-factor model.

647 Philos. Psychol. 24, 223-243 (2011). 
648 15. Borsboom, D., Mellenbergh, G. \& Van Heerden, J. The theoretical status of latent variables.

649 Psychol. Rev. 110, 203 (2003).

650 16. de Winter, J., Dodou, D. \& Stanton, N. A quarter of a century of the DBQ: some

651 supplementary notes on its validity with regard to accidents. Ergonomics 58, 1745-1769 (2015).

652 17. De Winter, J. Small sample sizes, overextraction, and unrealistic expectations: A

653 commentary on M. Mattsson. Accid. Anal. Prev. 50, 776-777 (2013).

654 18. de Winter, J. \& Dodou, D. The Driver Behaviour Questionnaire as a predictor of accidents: A 655 meta-analysis. J. Saf. Res. 41, 463-470 (2010).

656 19. Parker, D., Reason, J., Manstead, A. \& Stradling, S. Driving errors, driving violations and

657 accident involvement. Ergonomics 38, 1036-1048 (1995).

658 20. Rowe, R., Roman, G., McKenna, F., Barker, E. \& Poulter, D. Measuring errors and

659 violations on the road: A bifactor modeling approach to the Driver Behavior Questionnaire.

660 Accid. Anal. Prev. 74, 118-125 (2015).

661 21. Stanojević, P., Lajunen, T., Jovanović, D., Sârbescu, P. \& Kostadinov, S. The driver

662 behaviour questionnaire in South-East Europe countries: Bulgaria, Romania and Serbia. Transp.

663 Res. Part F Traffic Psychol. Behav. 53, 24-33 (2018).

664 22. Mattsson, M., Lajunen, T., Gormley, M. \& Summala, H. Measurement invariance of the

665 Driver Behavior Questionnaire across samples of young drivers from Finland and Ireland. Accid.

666 Anal. Prev. 78, 185-200 (2015).

667 23. Epskamp, S. \& Fried, E. A tutorial on regularized partial correlation networks. Psychol.

668 Methods Advance online publication available at http://psycnet.apa.org/record/2018-13501-001

669 (2018).

670 24. Kossakowski, J., Epskamp, S., Kieffer, J., van Borkulo, C., Rhemtulla, M. \& Borsboom, D.

671 The application of a network approach to Health-Related Quality of Life (HRQoL): introducing

672 a new method for assessing HRQoL in healthy adults and cancer patients. Quality of Life

673 Research 25, 781-792 (2016).

674 25. Golino, H. \& Epskamp, S. Exploratory graph analysis: A new approach for estimating the

675 number of dimensions in psychological research. PloS one 12, e0174035 (2017).

676 26. Costantini, G., Richetin, J., Preti, E., Casini, E., Epskamp, S. \& Perugini, M. Stability and

677 variability of personality networks. A tutorial on recent developments in network psychometrics.

678 Pers. Individ. Dif. (2017).

679 27. Wells, P., Tong, S., Grayson, G. \& Jones, E. Cohort II-A study of learner and new drivers-

680 volume 1-main report, volume 2-questionnaires and data tables. Road Safety Research Report

681 (2008).

682 28. Roman, G., Poulter, D., Barker, E., McKenna, F. \& Rowe, R. Novice drivers' individual

683 trajectories of driver behavior over the first three years of driving. Accid. Anal. Prev. 82, 61-69

684 (2015).

685 29. Transport Research Laboratory. Safety, Security and Investigations Division \& Poulter, D.

686 Cohort II: a Study of Learner and Novice Drivers, 2001-2005. 2nd Edition. UK Data Service.

687 SN: 5985, http://doi.org/10.5255/UKDA-SN-5985-2 (2015).

688 30. Mattsson, M. On testing factorial invariance: A reply to J.C.F. de Winter. Accid. Anal. Prev.

689 63, 89-93 (2014).

690 31. Epskamp, S., Waldorp, L., Mõttus, R. \& Borsboom, D. The Gaussian graphical model in

691 cross-sectional and time-series data. Multivar. Behav. Res. 1-28 (2018),

692 https://doi.org/10.1080/00273171.2018.1454823. 
693 32. Shiffman, S., Stone, A. \& Hufford, M. Ecological momentary assessment.

694 Annu.Rev.Clin.Psychol. 4, 1-32 (2008).

695 33. Lauritzen, S. Graphical models (Clarendon Press, 1996).

696 34. R Core Team: A language and environment for statistical computing. Available at

697 https://www.R-project.org/ (2017).

698 35. Epskamp, S., Cramer, A., Waldorp, L., Schmittmann, V. \& Borsboom, D. qgraph: Network

699 visualizations of relationships in psychometric data. J. Stat. Softw. 48, 1-18 (2012).

700 36. Epskamp, S., Borsboom, D. \& Fried, E. Estimating psychological networks and their

701 accuracy: A tutorial paper. Behav. Res. Methods, 1-18 (2017).

702 37. Friedman, J., Hastie, T. \& Tibshirani, R. Sparse inverse covariance estimation with the

703 graphical lasso. Biostatistics 9, 432-441 (2008).

704 38. Foygel, R. \& Drton, M. Extended Bayesian information criteria for Gaussian graphical

705 models. Advances in neural information processing systems, 23 (2010).

706 39. Fruchterman, T. \& Reingold, E. Graph drawing by force-directed placement. Softw. Pract.

707 Exp. 21, 1129-1164 (1991).

708 40. Zhang, B. \& Horvath, S. A general framework for weighted gene co-expression network

709 analysis. Stat. Appl. Genet. Mol. Biol. 4 (2005).

710 41. Costantini, G. \& Perugini, M. Generalization of clustering coefficients to signed correlation

711 networks. PloS one 9, e88669 (2014).

712 42. Saramäki, J., Kivelä, M., Onnela, J., Kaski, K. \& Kertesz, J. Generalizations of the clustering

713 coefficient to weighted complex networks. Phys. Rev. E 75, 027105 (2007).

714 43. Chapman, B., Weiss, A. \& Duberstein, P. Statistical learning theory for high dimensional

715 prediction: Application to criterion-keyed scale development. Psychol. Methods 21, 603 (2016).

716 44. Friedman, J., Hastie, T. \& Tibshirani, R. Regularization Paths for Generalized Linear Models

717 via Coordinate Descent. J. Stat. Softw 33, 1-22 (2010).

718 45. Zou, H. \& Hastie, T. Regularization and variable selection via the elastic net. J. R. Stat. Soc.

719 Series B Stat. Methodol. 67, 301-320 (2005).

720 46. Mittlböck, M. \& Schemper, M. Explained variation for logistic regression. Stat. Med. 15,

721 1987-1997 (1996).

722 47. Borsboom, D. A network theory of mental disorders. World Psychiatry 16, 5-13 (2017).

723 48. Fuller, R. Towards a general theory of driver behaviour. Accid. Anal. Prev. 37, 461-472

724 (2005).

725 49. Summala, H. Towards understanding motivational and emotional factors in driver behaviour:

726 Comfort through satisficing in Modelling driver behaviour in automotive environments, 189-207

727 (Springer, 2007).

728 50. Lawton, R., Parker, D., Stradling, S. \& Manstead, A. Self-reported attitude towards speeding

729 and its possible consequences in five different road contexts. J. Community Appl. Soc. Psychol.

730 7, 153-165 (1997).

731 51. Parker, D., Manstead, A., Stradling, S., Reason, J. \& Baxter, J. Intention to commit driving

732 violations: An application of the theory of planned behavior. J. Appl. Psychol. 77, 94 (1992).

733 52. Warner, H. \& Åberg, L. Drivers' decision to speed: A study inspired by the theory of planned

734 behavior. Transp. Res. Part F Traffic Psychol. Behav. 9, 427-433 (2006).

735 53. Abdel-Aty, M. \& Radwan, A. Modeling traffic accident occurrence and involvement. Accid.

736 Anal. Prev. 32, 633-642 (2000).

737 54. Stanton, N. \& Salmon, P. Human error taxonomies applied to driving: A generic driver error

738 taxonomy and its implications for intelligent transport systems. Saf. Sci. 47, 227-237 (2009). 
739

740

741

742

743

744

745

746

747

748

749

750

751

752

753

754

755

756

757

758

759

760

761

762

763

764

765

766

767

768

769

770

771

772

773

774

775

776

777

778

55. Björklund, G. Driver irritation and aggressive behaviour. Accid. Anal. Prev. 40, 1069-1077 (2008).

56. Cramer, A., van der Sluis, S., Noordhof, A., Wichers, M., Geschwind, N., Aggen, S., Kendler, K. \& Borsboom, D.Dimensions of normal personality as networks in search of equilibrium: You can't like parties if you don't like people. Eur. J. Pers. 26, 414-431 (2012). 57. Mischel, W. \& Shoda, Y. Reconciling processing dynamics and personality dispositions. Annu. Rev. Psychol. 49, 229-258 (1998).

58. Maasalo, I., Lehtonen, E. \& Summala, H. Young females at risk while driving with a small child. Accid. Anal. Prev. 108, 321-331 (2017).

59. Warner, H., Özkan, T., Lajunen, T. \& Tzamalouka, G. Cross-cultural comparison of drivers' tendency to commit different aberrant driving behaviours. Transp. Res. Part F Traffic Psychol. Behav. 14, 390-399 (2011).

60. Epskamp, S. Network psychometrics. D. Phil. Thesis, University of Amsterdam, available at http://sachaepskamp.com/Dissertation (2017).

61. Robinson-Cimpian, J. Inaccurate estimation of disparities due to mischievous responders: Several suggestions to assess conclusions. Educational Researcher 43, 171-185 (2014). 62. Precht, L., Keinath, A. \& Krems, J. Identifying effects of driving and secondary task demands, passenger presence, and driver characteristics on driving errors and traffic violationsUsing naturalistic driving data segments preceding both safety critical events and matched baselines. Transp. Res. Part F Traffic Psychol. Behav. 51, 103-144 (2017). 63. Fastenmeier, W. \& Gstalter, H. Driving task analysis as a tool in traffic safety research and practice. Saf. Sci. 45, 952-979 (2007). 64. Oppenheim, I. \& Shinar, D. A context-sensitive model of driving behaviour and its implications for in-vehicle safety systems. Cogn. Tehcnol. Work 14, 261-281 (2012). 65. Epskamp, S., Rhemtulla, M., \& Borsboom, D. Generalized network psychometrics: Combining network and latent variable models. Psychometrika, 82(4), 904-927 (2017). 66. Epskamp, S., Kruis, J., \& Marsman, M. Estimating psychopathological networks: Be careful what you wish for. PloS one, 12(6), e0179891 (2017).

67. Epskamp, S., Waldorp, L., Mõttus, R., \& Borsboom, D. The Gaussian Graphical Model in Cross-Sectional and Time-Series Data. Multivariate behavioral research, 1-28 (2018). 68. Embretson, S. Applications of cognitive design systems to test development. In Cognitive assessment, pp. 107-135. (Springer, Boston, MA, 1994). 69. Kline, R. Principles and practice of structural equation modeling ( $3^{\text {rd }}$ ed.). (Guilford publications, New York, 2011).

70. Schmittmann, V., Cramer, A., Waldorp, L., Epskamp, S., Kievit, R., \& Borsboom, D. Deconstructing the construct: A network perspective on psychological phenomena. New ideas in psychology, 31(1), 43-53 (2013).

71. Cramer, A., van der Sluis, S., Noordhof, A., Wichers, M., Geschwind, N., Aggen, S., Kendler, K. \& Borsboom, D. Measurable like temperature or mereological like flocking? On the nature of personality traits. European Journal of Personality, 26(4), 451-459 (2012). 


\section{Table 1 (on next page)}

Regression model fit in the training and hold-out samples 


\begin{tabular}{lccc} 
Variable & Elastic net & Ridge & Naive Poisson \\
\hline Training sample & & & \\
Pearson r & 0.246 & 0.260 & 0.317 \\
Mean Square Error & 0.625 & 0.622 & 0.590 \\
Min-Max Index & 0.121 & 0.122 & 0.128 \\
McFadden Pseudo R ${ }^{2}$ & 0.030 & 0.033 & 0.057 \\
Deviance & 968.4 & 964.8 & 924.8 \\
Null model deviance & & 1018.1 & \\
Hold-out sample & & & \\
Pearson r & 0.155 & 0.160 & 0.086 \\
Mean Square Error & 0.627 & 0.626 & 0.745 \\
Min-Max Index & 0.158 & 0.157 & 0.156 \\
McFadden Pseudo R ${ }^{2}$ & 0.010 & 0.011 & -0.044 \\
Deviance & 317.9 & 317.2 & 348.4 \\
Null model deviance & & 323.6 & \\
\hline
\end{tabular}

1 


\section{Table 2 (on next page)}

Regression weights of predicting accidents based on the elastic net regression analysis 


\begin{tabular}{|c|c|}
\hline Variable & Regression weight \\
\hline Mileage & 0.04 \\
\hline \multicolumn{2}{|l|}{ Gender } \\
\hline \multicolumn{2}{|l|}{ Age } \\
\hline Drive away from traffic lights at too high a gear & 0.07 \\
\hline Overtake a slow driver on inside & 0.04 \\
\hline \multicolumn{2}{|l|}{ Have to confirm you're in right gear } \\
\hline \multicolumn{2}{|l|}{ Attempt to overtake and hadn't noticed signalling right } \\
\hline \multicolumn{2}{|l|}{ Forget where left car in carpark } \\
\hline Sound horn to indicate annoyance & 0.07 \\
\hline \multicolumn{2}{|l|}{ Switch on one thing when meant to switch on other } \\
\hline \multicolumn{2}{|l|}{ Change into wrong gear when driving along } \\
\hline Pull out of junction so far that driver has to let you out & 0.04 \\
\hline \multicolumn{2}{|l|}{ Have used plates to warn drivers you are a new driver } \\
\hline \multicolumn{2}{|l|}{ Realised have no recollection of road been travelling } \\
\hline Crossed junction knowing lights have turned against you & 0.01 \\
\hline Failed to notice people crossing when turned into side street & 0.08 \\
\hline \multicolumn{2}{|l|}{ Become angered by driver and given chase } \\
\hline \multicolumn{2}{|l|}{ Misread signs and taken wrong turning off roundabout } \\
\hline \multicolumn{2}{|l|}{ Drive in either too low or high gear for conditions } \\
\hline Disregard speed limit on residential road & 0.04 \\
\hline \multicolumn{2}{|l|}{ When turning left have nearly hit cyclist on inside } \\
\hline \multicolumn{2}{|l|}{ Used mobile phone without hands free kit } \\
\hline \multicolumn{2}{|l|}{ Stay in motorway lane know will be closed } \\
\hline \multicolumn{2}{|l|}{ When queueing to turn left nearly hit car in front } \\
\hline \multicolumn{2}{|l|}{ Drive when suspect over legal alcohol limit } \\
\hline Forget to take handbrake off before moving off & 0.03 \\
\hline Become angered by driver and indicate hostility & 0.06 \\
\hline \multicolumn{2}{|l|}{ Misjudge speed of oncoming vehicle when overtaking } \\
\hline Hit something when reversing that hadn't seen & 0.06 \\
\hline Raced away from traffic lights to beat other driver & 0.01 \\
\hline \multicolumn{2}{|l|}{ Used a hands free kit } \\
\hline \multicolumn{2}{|l|}{ Selected wrong gear when wanting to go in reverse } \\
\hline Noticed on different road to destination want to go & -0.01 \\
\hline \multicolumn{2}{|l|}{ Get into wrong lane when approaching roundabout/junction } \\
\hline \multicolumn{2}{|l|}{ Drive so close to car that wouldn't be able to stop } \\
\hline \multicolumn{2}{|l|}{ Forget headlights were on full beam } \\
\hline Missed give-way signs and barely avoided collision & 0.02 \\
\hline & 0.01 \\
\hline \multicolumn{2}{|l|}{ Failed to check rear-view mirror before manoeuvring } \\
\hline \multicolumn{2}{|l|}{ Brake too quickly on slippery road or steer wrong in skid } \\
\hline \multicolumn{2}{|l|}{ Drove after taking drugs which think affected you } \\
\hline Brake/swerve to avoid accident & 0.01 \\
\hline
\end{tabular}


Figure 1 (on next page)

The latent variable view of violations and errors.

Rectangles refer to observed variables, circles and ovals to latent variables. The ovals with the letter " $\mathrm{e}$ " and a number refer to error variances of the respective observed variables. The error variances (e1-e7) are assumed uncorrelated as per the assumption of conditional independence. Arrows pointing in one direction refer to assumed causal associations, while the two-headed arrow represents a covariance relation. To simplify the figure, predictors for errors are not shown 


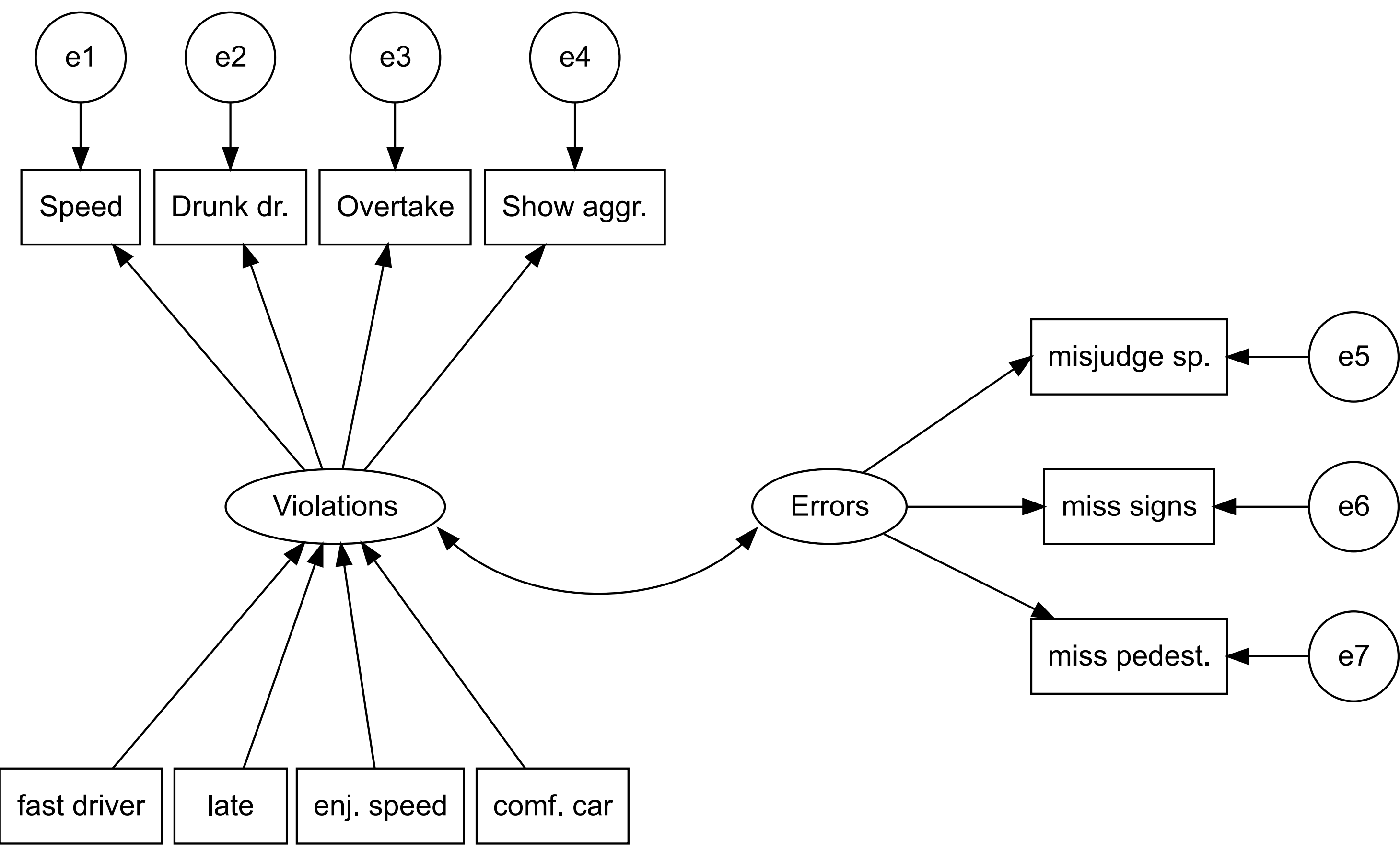


Figure 2 (on next page)

The network view of traffic behavior.

Traffic behaviors encoded in the DBQ are drawn using solid lines, background factors using dashed lines. Pairwise relationship among individual violations and errors, when controlling for the effects of all other variables, are shown as the edges of the network. The background variables "congestion" and "stress" that were not included in Figure 1 have been added to indicate the possibility that there exist background variables that are related to only one of the driver behaviors included in the model 


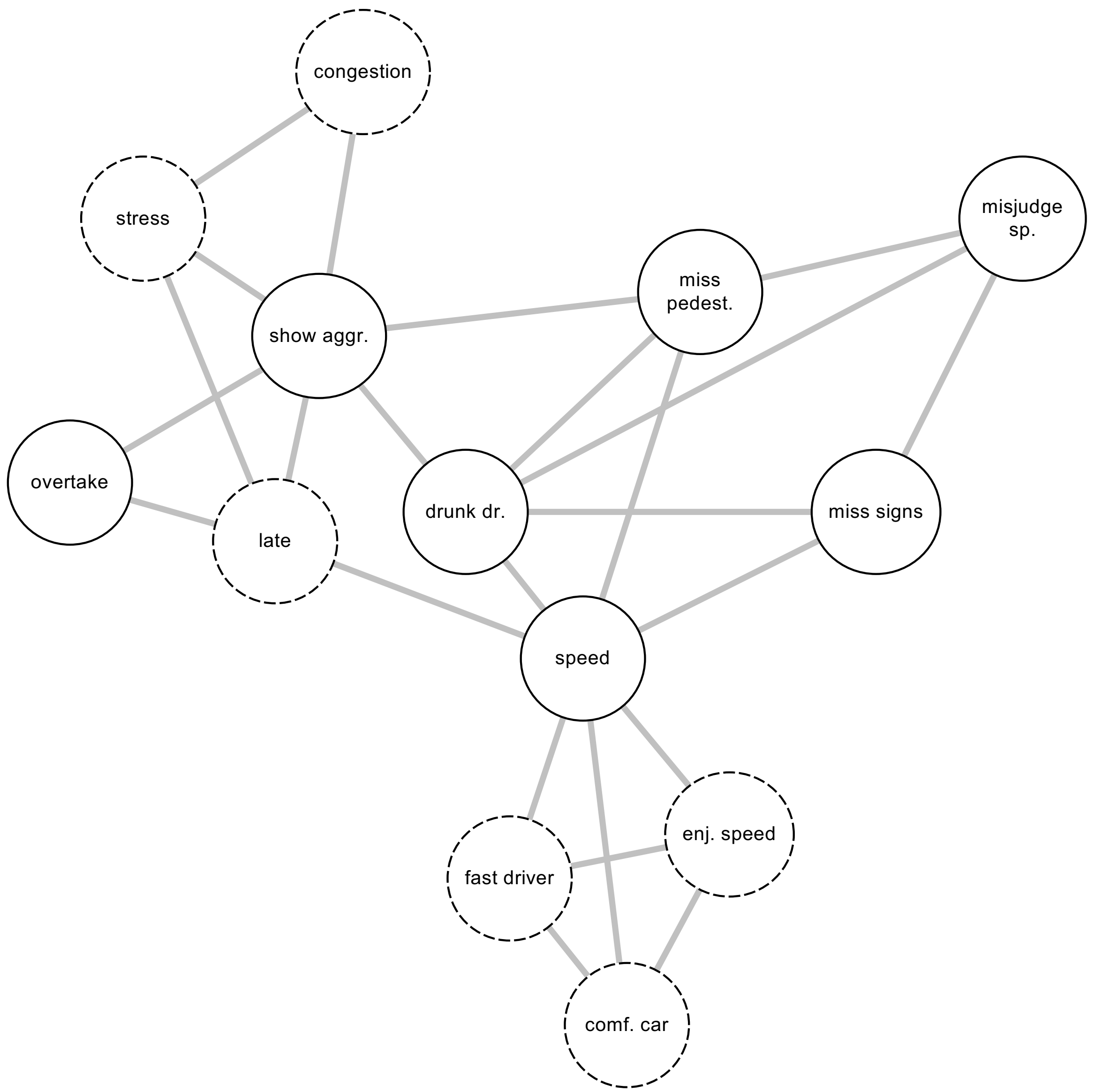




\section{Figure 3 (on next page)}

The between-person network model

The colors of the nodes correspond with the errors / violations dichotomy commonly used in DBQ studies. Green edges signify positive associations, dashed red edges negative associations. The wider and the more opaque the edge, the stronger the association. 


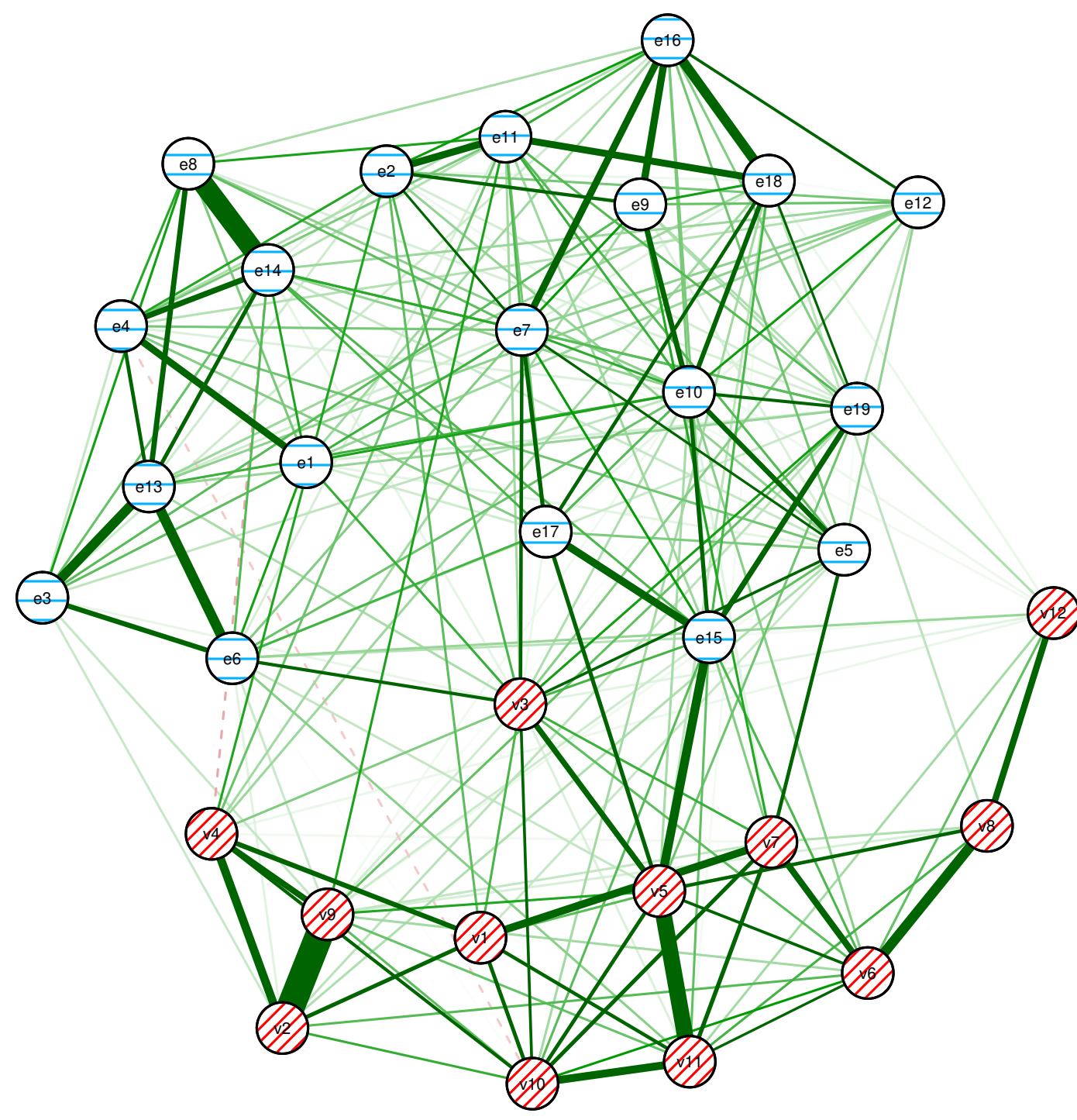

\section{Error}

- e1: drive away from traffic lights at too high a gear

0 e2: attempt to overtake and hadn't noticed signalling right

- e3: forget where left car in carpark

- e4: switch on one thing when meant to switch on other

- e5: pull out of junction so far that driver has to let you out

- e6: realised have no recollection of road been travelling

- e7: failed to notice people crossing when turned into sidestreet

- e8: misread signs and taken wrong turning off roundabout

- e9: turning left, nearly hit cyclist on inside

- e10: when queuing to turn left nearly hit car in fron

- e11: misjudged speed of oncoming vehicle when overtaking

- e12: hit something when reversing that hadn't seen

- e13: noticed ending up on a different road than intended

- e14: get into wrong lane when approaching roundabout/junction

- e15: drive so close to car that would not be able to stop

- e16: missed giveway signs and avoided colliding with traffic

- e17: failed to check rear-view mirror before manoeuvring

- e18: brake too quickly on slippery road or steer wrong in skid

- e19: had to brake or swerve to avoid accident

\section{Violation}

- v1: overtake a slow driver on inside

- v2: sound horn to indicate annoyance

- v3: crossed junction knowing lights have turned against you

- v4: become angered by driver and given chase

- v5: disregarded speed limit on residential road

- v6: used mobile phone without hands free kit

- v7: stay in motorway lane you know will be closed

- v8: drive when suspect over legal alcohol limit

- v9: become angered by driver and indicated hostility

- v10: raced away from traffic lights to beat other driver

- v11: disregarded speed limit on motorway

- v12: drove after taking drugs which affected you 
Figure 4 (on next page)

Indices of centrality and clustering for the between-person network. 
A Closeness

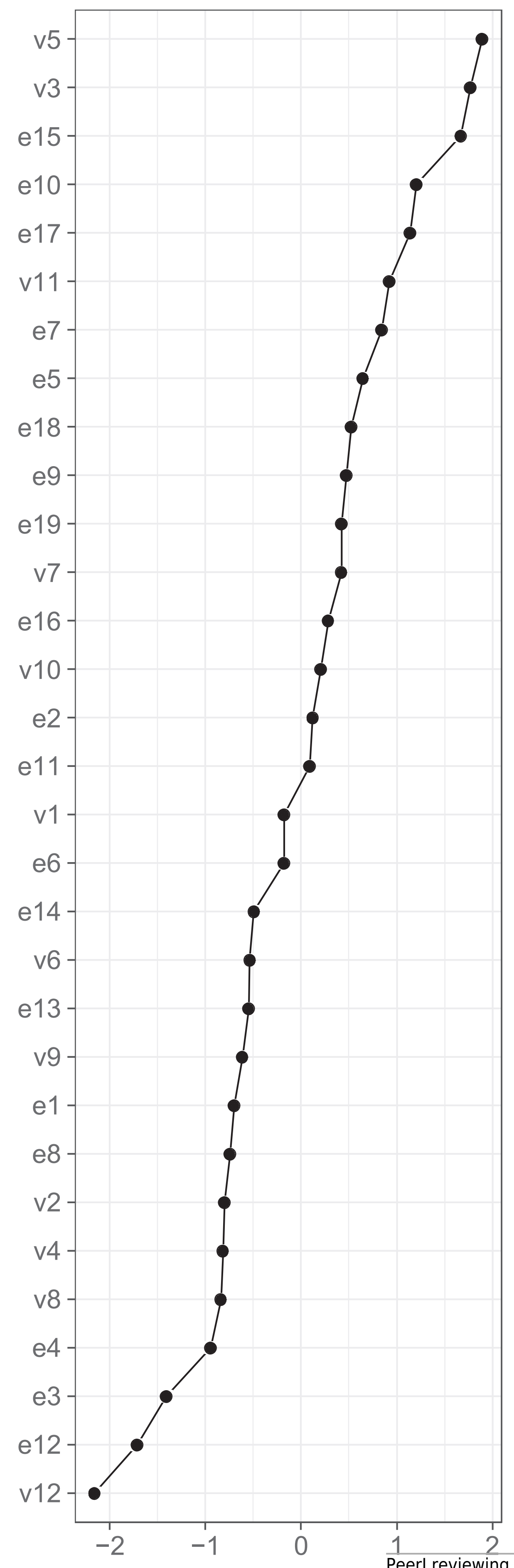

B

Strength Manuscript to b $\mathcal{E}_{\text {reviedclustering coefficient }}$
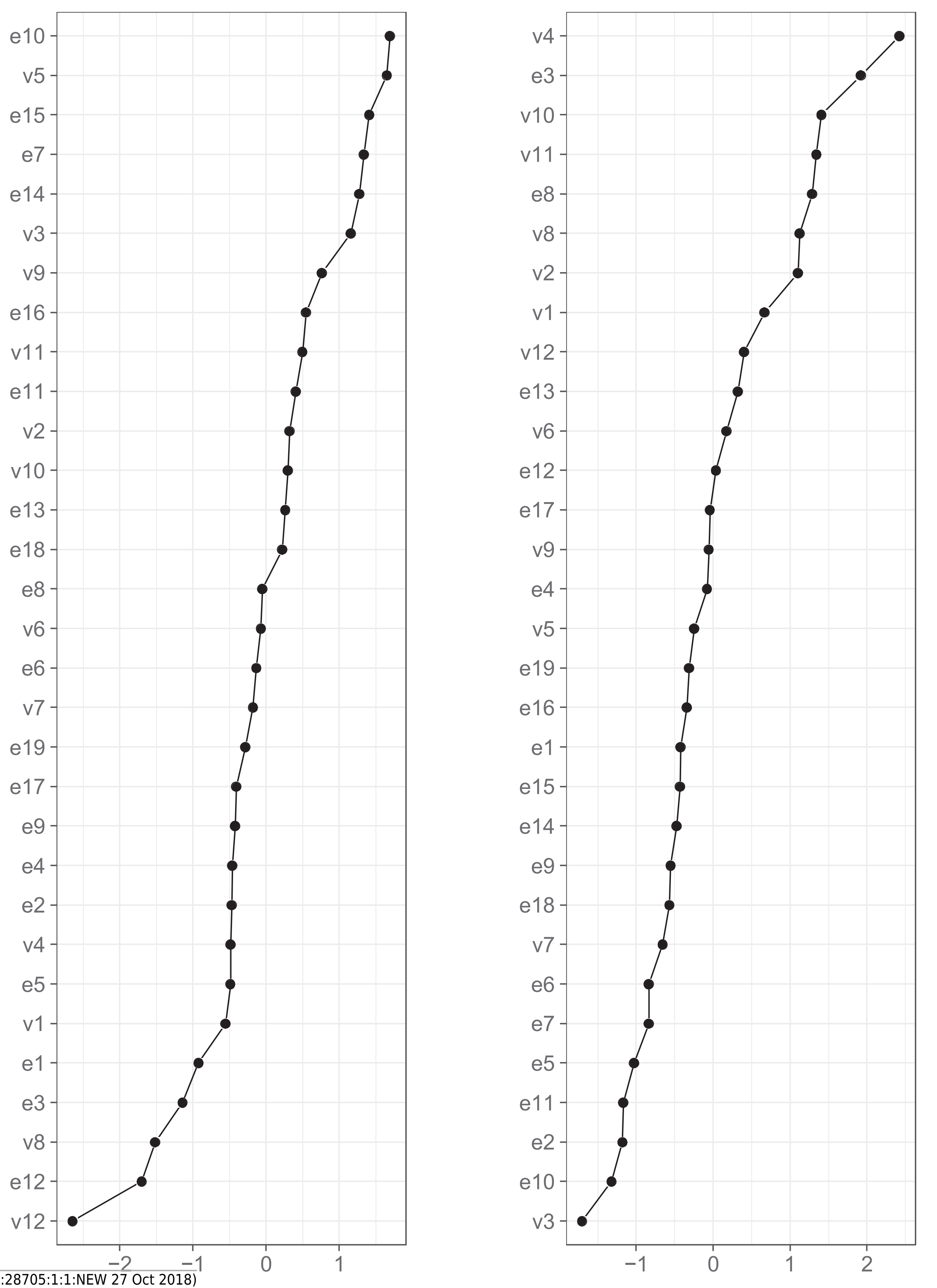


\section{Figure $\mathbf{5}$ (on next page)}

The cross-sectional network model at the first time point ( 6 months post-licensure) showing the relationships of the DBQ variables and background variables. 


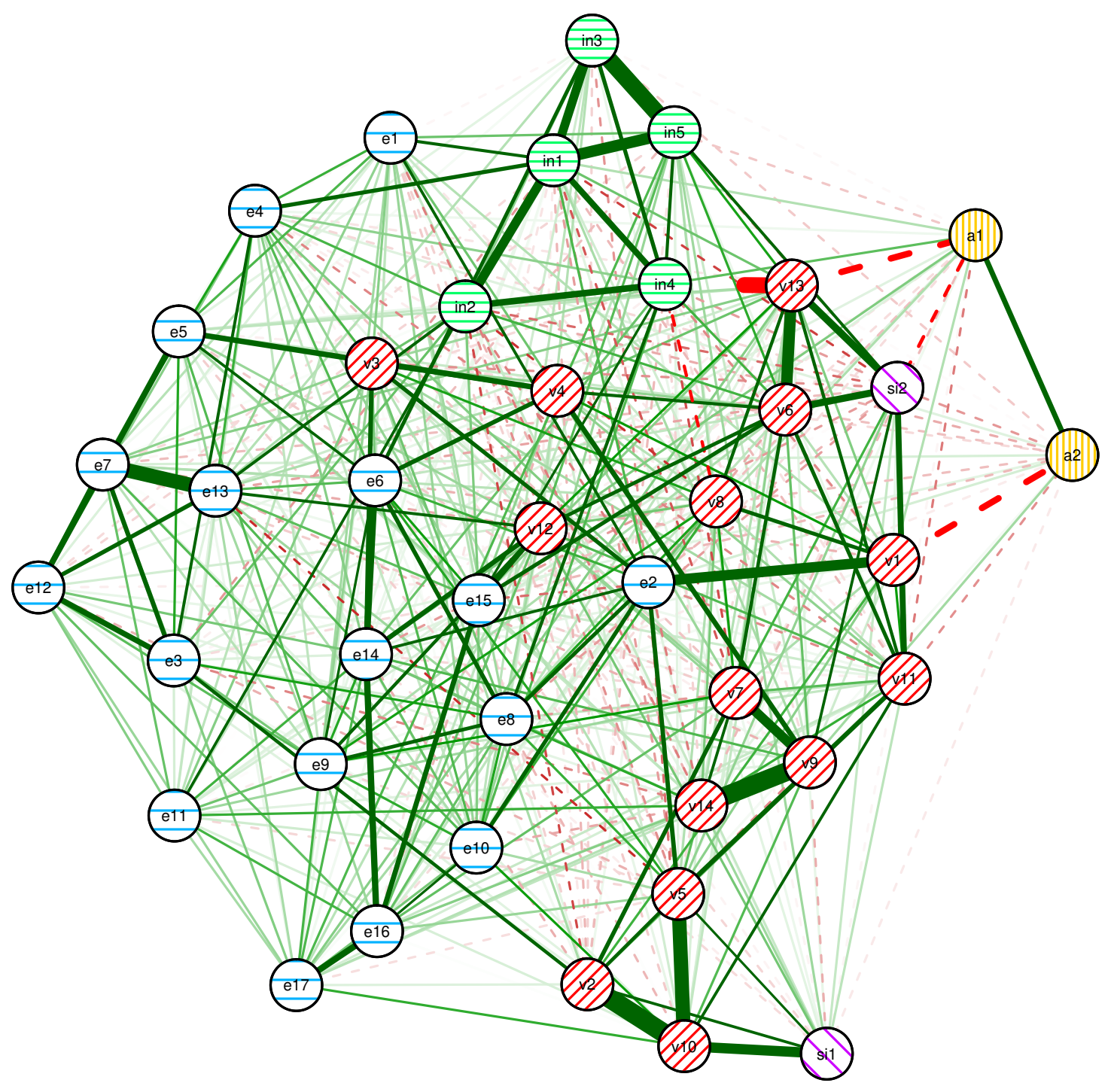

Attitude

- a1: Decreasing motorway speed limit is a good idea

- a2: Cars should never overtake on inside lane

Error

- e1: drive away from traffic lights at too high a gear - e2: attempt to overtake and hadn't noticed signalling right e3: forget where left car in carpark

- e4: switch on one thing when meant to switch on other

- e6: failed to notice people crossing when turned into sidestreet

- e: misread signs and taken wrong turning off roundabout

- e8: turning left, nearly hit cyclist on inside

- e9: when queuing to turn left nearly hit car in front

- e10: misjudged speed of oncoming vehicle when overtaking

- e11: hit something when reversing that hadn't seen

- e12: noticed ending up on a different road than intended

- e13: get into wrong lane when approaching roundabout/junction

- e14: missed giveway signs and avoided colliding with traffic

- e15: falled to check rear-view mirror before manoeuvring

- e16: brake too quickly on slippery road or steer wrong in skid

- e17: had to brake or swerve to avoid accident

Improvement needs

- in1: improve car contro

O in2: improve parking

- ins: improve on changing laards

D in5: improve on knowing what speed is safe

Self-image

- si1: Kind of driver - irritable/placid

- si2: Kind of driver - slow/fas

Violation

- v1: overtake a slow driver on inside

- v2: sound horn to indicate annoyance

- v3. pull out of junction so far hat driver has th

- v4: crossed junction knowing lights have turned against you

- v5: become angered by driver and given chase

- v6: disregarded speed limit on residential road

- v7: used mobile phone without hands free kit

- v8: stay in motorway lane you know will be clo

- vio: become angeed by drive and indicated ho

- v11: raced away from traffic lights to beat other drivity

- v12: drive so close to car that would not be able to stop

- v13: disregarded speed limit on motorway

- v14: drove after taking drugs which affected you 


\section{Figure $\mathbf{6}$ (on next page)}

Indices of centrality and clustering for the cross-sectional network. 
A

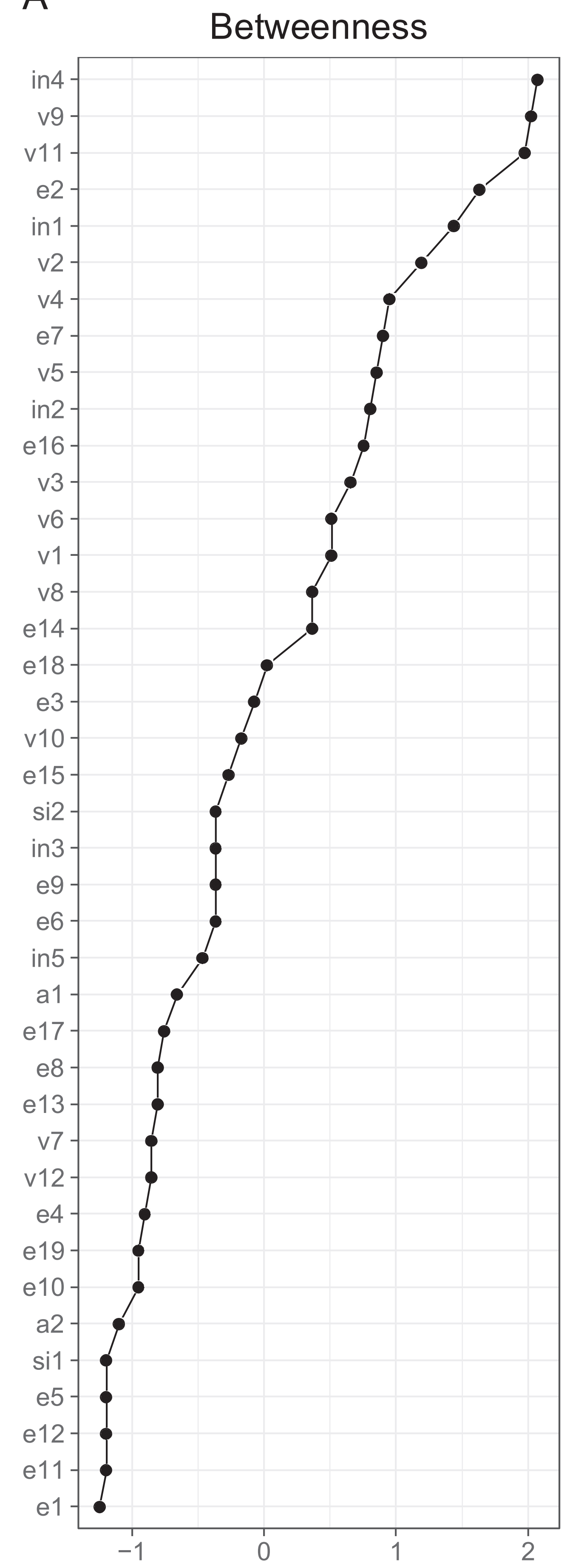

B PeerJ

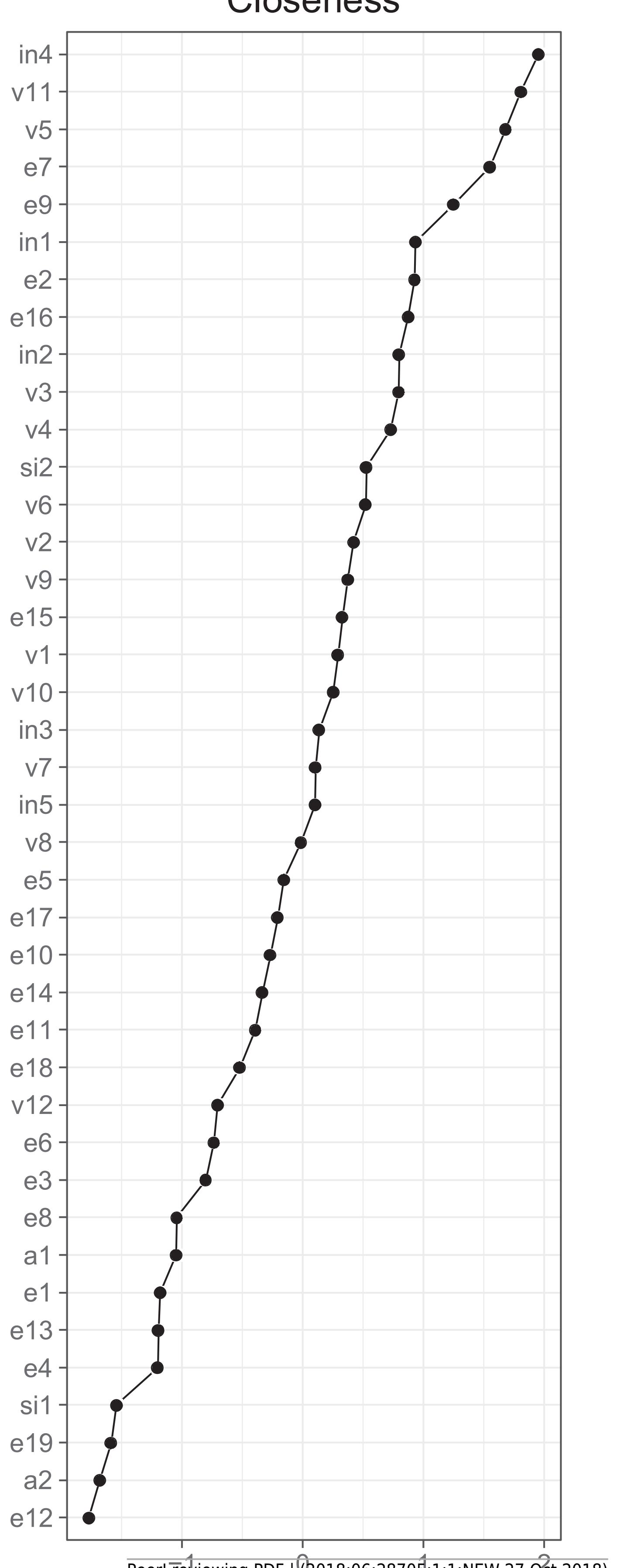

C Manuscriptsterengithviewed

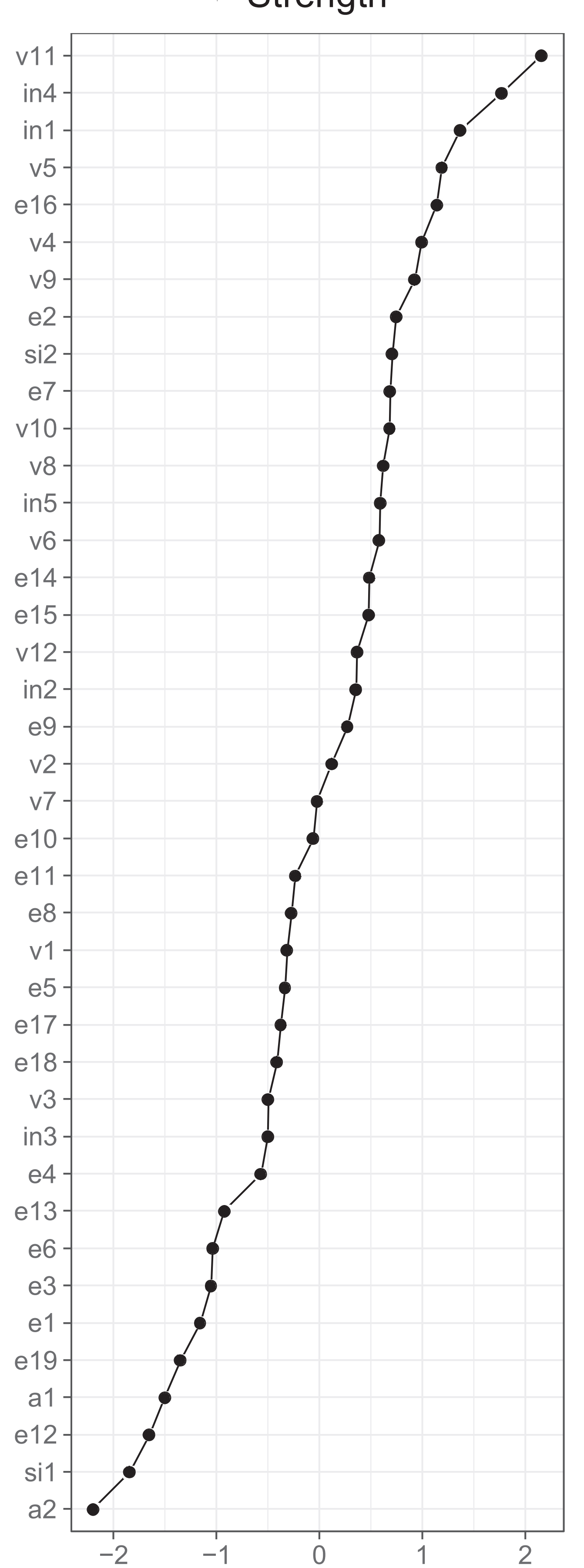

Clustering coefficient

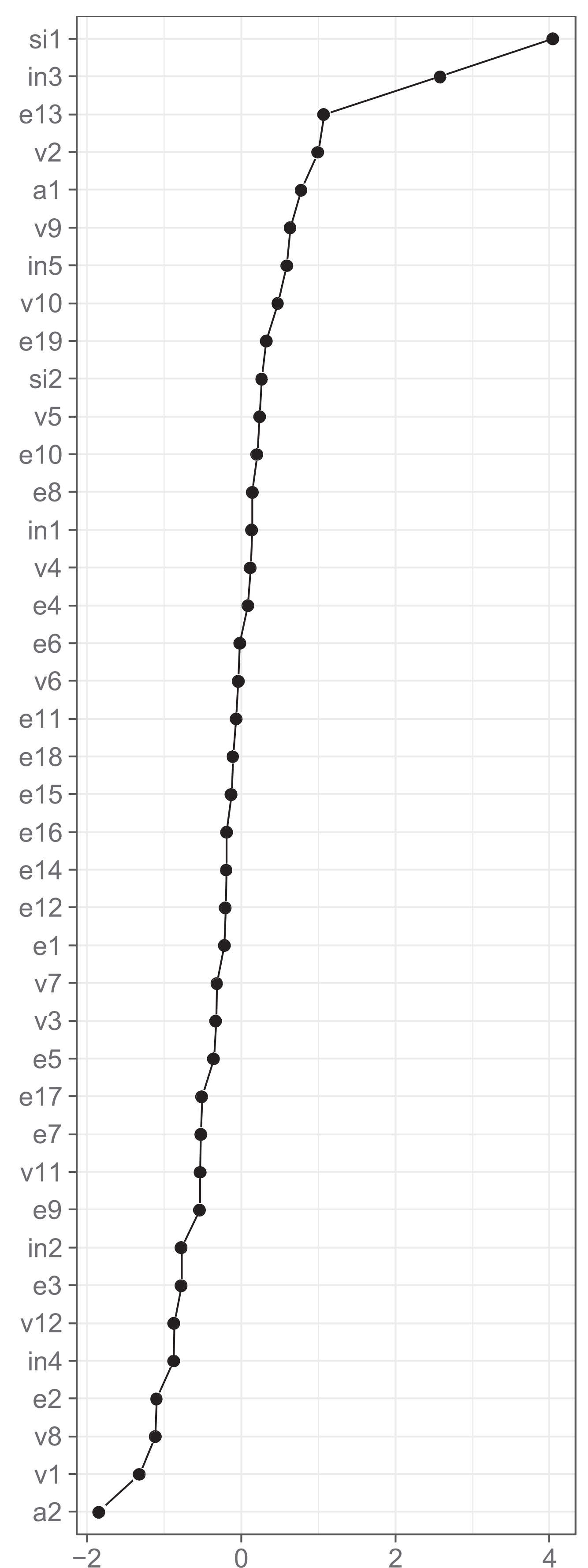

